\title{
Características biológicas e morfológicas de cepas brasileiras de Schistosoma mansoni em Mus musculus
}

\author{
Parasitological and morphological characteristics of Brazilian strains \\ of Schistosoma mansoni in Mus musculus
}

\author{
Elaine Machado Martinez', Renata Heisler Neves², Regina Maria Figueiredo de Oliveira², \\ José Roberto Machado-Silva ${ }^{2}$ e Luís Rey ${ }^{3}$
}

\begin{abstract}
Resumo A fim de verificar diferenças biológicas e morfológicas entre cepas brasileiras (CMO, CM e BE) de Schistosoma mansoni foram estudados os seguintes parâmetros: período pré-patente, cinética de eliminação de ovos nas fezes, contagem de ovos no intestino, infectividade e as características fenotípicas dos vermes adultos. O período pré-patente foi de 42 a 44 dias. A recuperação de vermes adultos variou de $26 \%$ a $29 \%$, sem diferenças significativas $(p>0,05)$ nestes parâmetros. Todas as cepas apresentaram maior quantidade de ovos no intestino delgado do que no grosso $(p<0,05)$. Diferenças significativas foram observadas no aparelho reprodutor e ventosas dos adultos machos. Todas as medidas foram menores na cepa CMO. As fêmeas apresentaram diferenças significativas no maior diâmetro do ovo, área e perímetro do espinho do ovo e na área da ventosa oral. Concluímos que as diferenças entre cepas podem ser expressas mesmo quando estas são mantidas por várias gerações em condições de laboratório.
\end{abstract}

Palavras-chaves:Schistosoma mansoni. Cepas. Estudo parasitológico. Morfologia.

Abstract Aiming to determine parasitological and morphological differences between Brazilian strains (CMO, $C M$ and $B E$ ) of Schistosoma mansoni the following parameters were studied: prepatent period, kinetics of egg releasing in feces, intestinal oogram, infectivity and the morphology of adult worms. No statistical difference ( $p>0.05$ ) was found regarding the mean prepatent period (44 and 46 days) and infectivity (26\% and $29 \%$ ). All strains showed a higher concentration of trapped eggs in the small intestine than large intestine $(p<0.05)$. All characteristics of the reproductive system and suckers of male worms presented significant differences $(p<0.05)$. $C M O$ strain was smaller in all measurements. Females showed significant differences $(p<0.05)$ in the larger diameter of the eggs, in the area and perimeter of the spine egg and in the oral sucker area. We conclude that differences in strains can be expressed even when the strains have been cycled for several generations under laboratory conditions.

Key-words: Schistosoma mansoni. Strains. Parasitological study. Morphology. Mus musculus.

O parasitismo humano por Schistosoma mansoni é decorrente de uma linhagem de parasitos que evoluiu anteriormente em roedores ${ }^{13}$. Admite-se que esta espécie tenha surgido na Ásia, migrado para a África e atingido a região neotropical durante o tráfico de escravos $^{52}$. Ao longo deste processo evolutivo, foram selecionadas subpopulações (cepas) devido ao desenvolvimento do helminto em diversas ordens de vertebrados e espécies de moluscos da família Planorbidae 2144 .

Kloetzel26 questionou a existência de cepas no Brasil no final dos anos 50. Este questionamento foi esclarecido ao se demonstrar a existência de especificidade entre 0 parasito e diferentes espécies de Biomphalaria ${ }^{39} 40$.

\footnotetext{
1. Departamento de Biologia Parasitária do Instituto Oswaldo Cruz da Fundação Oswaldo Cruz, Rio de Janeiro, RJ. 2. Curso de Biologia Parasitária do Departamento de Patologia e Laboratórios da Faculdade de Ciências Médicas da Universidade do Estado do Rio de Janeiro, Rio de Janeiro, RJ. 3. Departamento de Medicina Tropical da Fundação Oswaldo Cruz, Rio de Janeiro, RJ.

Endereço para correspondência: Dr. José Roberto Machado e Silva. Curso de Biologia Parasitária/Depto de Patologia e Laboratórios/FCM/UERJ.

R. Prof. Manoel de Abreu 48/5ํandar, Vila Isabel, 20551-170 Rio de Janeiro, RJ, Brasil.

Telefax: $55212587-6148$

e-mail: machado@uerj.br

Recebido para publicação em 15/4/2002

Aceito em 23/7/2003
} 
Os experimentos realizados em Mus musculus demonstraram que as cepas apresentavam diferenças no período pré-patente, infectividade, patogenicidade, cinética de eliminação de ovos nas fezes e contagem de ovos no fígado ou parede intestinal ${ }^{15} 16245355$. Diferenças também foram verificadas nos vermes adultos 293033374147 .

Diversos autores demonstraram que passagens sucessivas do $S$. mansoni em laboratório produzem diminuição da sua variabilidade genética, mudanças na infectividade para hospedeiros intermediários e para os definitivos 20284

O presente artigo relata um estudo feito, concomitantemente, sobre as características biológicas e morfológicas de vermes adultos de cepas brasileiras de $S$. mansoni mantidas há mais de cinco anos em condições de laboratório, a fim de verificar se esta manutenção prolongada afetava tais características.

\section{MATERIAL E MÉTODOS}

Parasitos. As cepas aqui estudadas foram originalmente isoladas em distintos estados: BE, a partir de Biomphalaria glabrata coletada naturalmente infectada no Bairro do Telégrafo no Município de Belém do Pará (Estado do Pará); $\mathrm{CMO}$, isolada a partir das fezes do roedor silvestre Oryzomys subflavus (Rodentia: Cricetidae) com infecção esquistossomótica natural no Município de Ceará-Mírim (Estado do Rio Grande do Norte) e CM obtida a partir de fezes humanas, no Engenho Pitangueiras no Município de São Lourenço da Mata (Estado de Pernambuco). Todas as cepas estão sendo mantidas por passagens sucessivas em Mus musculus Swiss Webster e Biomphalaria glabrata simpátrica, durante cinco (cepa $\mathrm{CM}$ ) ou vinte anos (cepas BE e CMO), no Departamento de Malacologia do Instituto Oswaldo Cruz.

Infecção experimental. Trinta e nove camundongos fêmeas, com 35 a 40 dias de idade, pesando 20 gramas, foram fornecidas pelo Centro de Criação de Animais de Laboratório (CECAL-FIOCRUZ, Rio de Janeiro, Brasil). Cada lote com 13 animais foi infectado por via subcutânea com 80 cercárias recém eliminadas por $B$. glabrata, infectada por uma das cepas acima mencionadas. Após a infecção, os animais foram mantidos em gaiolas de polipropileno $(40 \mathrm{~cm} \times 33 \mathrm{~cm})$ e alimentados com a ração industrializada convencional Nuvilab CR1 (município de Colombo, Estado do Paraná, Brasil) e água ad libitum. Os experimentos foram realizados de acordo com os procedimentos éticos com animais de experimentação ${ }^{56}$.

Estudo biológico. Eliminação de ovos nas fezes: entre 35 e 54 dias após a infecção, amostras de fezes de 24 horas foram recolhidas, individualmente, três vezes por semana. A análise parasitológica foi realizada pelo método de Kato-Katz ${ }^{25}$ e uma técnica por sedimentação quantitativa ${ }^{47}$. Em cada exame foram analisadas duas lâminas por animal. A viabilidade dos ovos foi aferida segundo movimentos do miracídio no interior do ovo ou atividade das células em flama.

Determinação da infectividade: após nove semanas de infecção, todos os animais foram sacrificados por deslocamento cervical, perfundidos com salina citratada a $0,9 \%$ e necropsiados ${ }^{50}$.

Distribuição quantitativa dos ovos nos segmentos intestinais: de cada animal, os intestinos delgado e grosso foram separados. O intestino delgado foi dividido em duas metades uma proximal e outra distal. Da porção anterior de cada segmento foi retirado $1 \mathrm{~cm}$ de comprimento.

Do segmento grosso, uma amostra foi retirada da porção justa-anal ${ }^{31}$. Os fragmentos foram digeridos em $5 \mathrm{ml}$ de hidróxido de potássio a $4 \%$ em banho-maria a $56^{\circ} \mathrm{C}$ por 120 minutos $^{10}$. Após a digestão, o material foi centrifugado a $2000 \mathrm{rpm}$ por três minutos. Ao sedimento foram acrescentados $0,5 \mathrm{ml}$ de água destilada e retiradas 5 alíquotas de $100 \mu \mathrm{l}$ para leitura em microscópio de luz, com aumento de 100 vezes. Como o tamanho dos segmentos foi determinado durante a necropsia, o número total de ovos foi estimado em cada segmento por regra aritmética, considerando-se o tamanho do segmento e o número de ovos contados na alíquota.

Estudo morfológico dos vermes adultos. Os vermes adultos foram fixados em AFA (álcool, formalina e ácido acético glacial), corados por carmim clorídrico, clarificados com salicilato de metila e montados em lâmina histológica com bálsamo do Canadá ${ }^{37}$.

O estudo morfométrico dos vermes adultos foi feito em um sistema computadorizado de análise de imagens (Image Pro Plus - Media Cybernetics) com análise dos seguintes caracteres dos lobos testiculares: número, área, maior e menor diâmetro e perímetro. No ovo uterino e o espinho foram estudados a área, maior e menor diâmetro e perímetro. Em ambos os sexos foram medidas as áreas das ventosas oral e ventral e a distância entre elas ${ }^{37}$. Foram analisados 150 espécimes machos: 60 (BE), $51(\mathrm{CM})$ e 39 (CMO); e 127 fêmeas: 49 (BE), 32 (CM) e 46 (CMO).

Análise estatística. Para os dados biológicos foram aplicados os testes de Mann-Whitney e de Regressão de Correlação linear ${ }^{49}$; e para os morfológicos, a Análise de Variância (One Way Anova). Valores de $\mathrm{p} \leq 0,05$ foram considerados significativos.

\section{RESULTADOS}

Entre os parâmetros analisados, foram verificadas diferenças significativas $(p=0,0001)$ apenas no percentual de distribuição de ovos do segmento grosso:
2\% (cepa CMO), 4\% (cepa CM) e 7\% (cepa BE). Todas as cepas apresentaram maior concentração de ovos (cerca de $80 \%$ ) no segmento distal (Figura 1). Através 
dos Testes de Regressão e Correlação Linear foi demonstrada uma correlação positiva ( $p=0,001$ e $r=0,848$ ) entre a contagem de ovos no intestino e o número total de fêmeas adultas e o total de ovos nas fezes ( $p=0,007$ e $r=0,731)$, apenas na cepa CMO.

Em todas as cepas, o período pré-patente foi de 42 (cepa CM) a 44 dias (cepas BE e CMO). A partir do $47^{\circ}$ dia de infecção houve um aumento progressivo na eliminação de ovos nas fezes até o último exame $\left(54^{\circ}\right.$ dia). Na cepa $B E$ foi pequeno o aumento (Figura 2). O número total de ovos eliminados nas fezes variou entre 1.872 (cepa CMO) e 6.408 (cepa CM). Embora a cepa CM tenha maior contagem de ovos, a viabilidade dos ovos foi menor (2\%) do que na CMO (38\%) e na BE (68\%).

Quanto a infectividade (percentual de recuperação de vermes adultos), não foram evidenciadas diferenças significativas ( $p=0,943)$ entre as cepas: CM

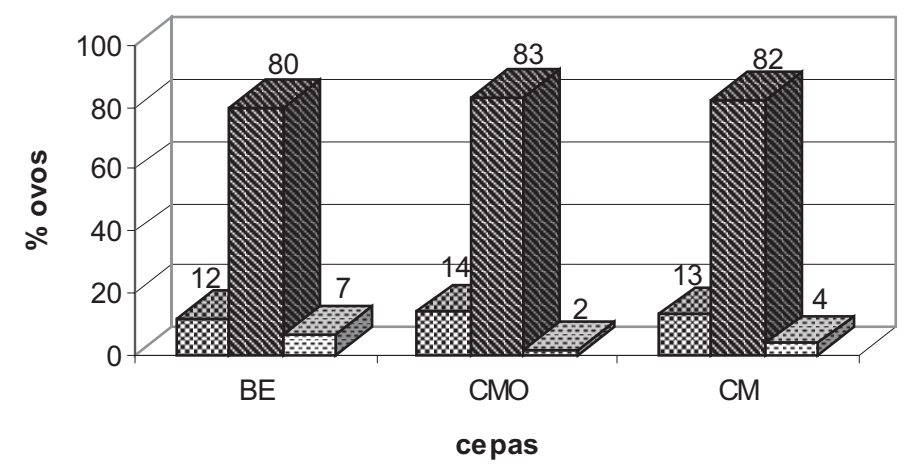

Proximal $\$$ Distal $⿴ 囗 ⿱ 一 一)$ Grosso

Figura 1 - Contagem de ovos (em percentual) no intestino delgado (segmentos proximal e distal) e no intestino grosso de camundongos albinos infectados com cepas brasileiras de Schistosoma mansoni albinos infectados
(BE, CM e CMO).

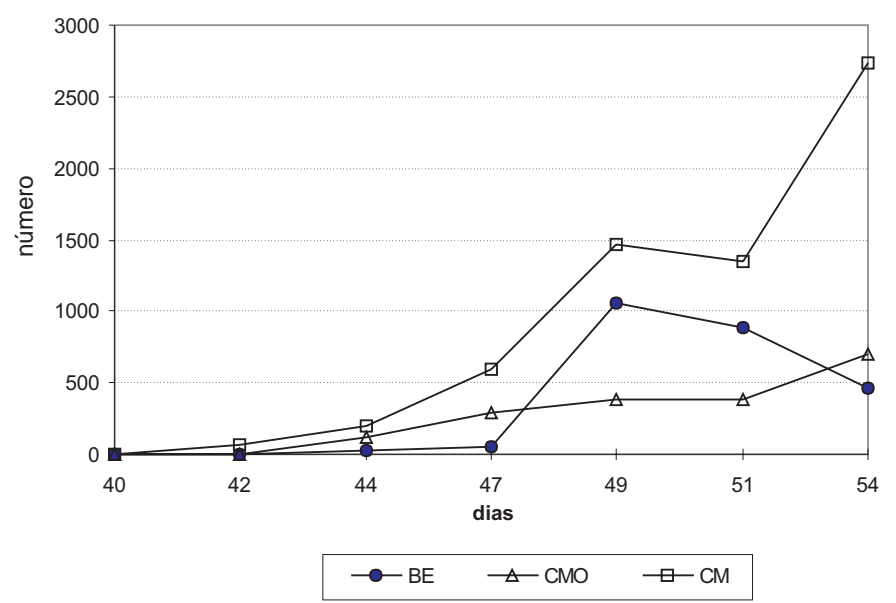

Figura 2- Cinética de eliminação de ovos nas fezes de camundongos albinos infectados com cepas brasileiras de Schistosoma manson (BE, CM e CMO), segundo o tempo de infecção (dias).

$(23,6 \pm 6,3 \%)$, CMO e BE $(20,8 \pm 6,0 \%)$. A proporção entre macho/fêmea foi de 1:1 (cepas BE e CM) e 0,9:1 (cepa CMO).

Os valores morfométricos dos espécimes machos estão discriminados na Tabela 1. Entre os cinco caracteres lobos testiculares analisados, todas as cepas apresentaram diferenças significativas $(p<0,05)$ na área, nos diâmetros e no perímetro. Também foram encontradas diferenças significativas na área da ventosa ventral. Todos os oito caracteres da cepa CMO 
eram menores do que nas demais cepas. A comparação entre os dados biométricos da cepa CMO com a cepa CM apontou diferenças significativas $(p<0,05)$. Apenas o número dos lobos testiculares e a distância entre as ventosas, não foram significativamente diferentes $(p>0,05)$ entre as cepas BE e CMO.

O diâmetro menor dos lobos testiculares, a área da ventosa oral e a distância entre as ventosas foram significativamente diferentes $(p<0,05)$ entre as cepas BE e CM. Apenas na cepa BE foram encontrados espécimes (4) com lobos testiculares supranumerários.

A análise morfométrica das fêmeas demonstrou diferenças significativas $(p<0,05)$ entre as cepas, porém em menor número de caracteres (Tabela 2). Nenhum dos caracteres estudados apresentou diferença significativa entre as três cepas. Diferenças significativas $(p<0,05)$ foram observadas na área e no perímetro do espinho (cepa $\mathrm{BE} \times \mathrm{CMO}$ ) e no maior diâmetro do ovo (cepa BE x CM). Entre as cepas CM e $\mathrm{CMO}$, as diferenças ficaram limitadas à área da ventosa oral.

\begin{tabular}{|c|c|c|c|}
\hline \multirow[t]{2}{*}{ Caracteres } & \multicolumn{3}{|c|}{ Cepas } \\
\hline & $\mathrm{BE}$ & $\mathrm{CM}$ & $\mathrm{CMO}$ \\
\hline \multicolumn{4}{|l|}{ Lobos testiculares } \\
\hline número ${ }^{1,2}$ & $7.8 \pm 2.0$ & $8.2 \pm 1.6$ & $7.1 \pm 1.4$ \\
\hline área $\left(\mu \mathrm{m}^{2}\right)^{1}$ & $25743 \pm 5975$ & $24109 \pm 5374$ & $12281 \pm 3781$ \\
\hline maior diâmetro $(\mu \mathrm{m})^{1}$ & $301 \pm 51$ & $291 \pm 53$ & $190 \pm 45$ \\
\hline menor diâmetro $(\mu \mathrm{m})^{2,3}$ & $103 \pm 17$ & $86 \pm 15$ & $71 \pm 14$ \\
\hline perímetro $(\mu \mathrm{m})^{1}$ & $733 \pm 127$ & $726 \pm 121$ & $469 \pm 94$ \\
\hline \multicolumn{4}{|l|}{ Ventosas (área) } \\
\hline oral $\left(\mu m^{2}\right)^{2,3}$ & $19411 \pm 5799$ & $23717 \pm 10173$ & $10209 \pm 5407$ \\
\hline ventral $\left(\mu \mathrm{m}^{2}\right)^{1}$ & $29639 \pm 10845$ & $32394 \pm 10873$ & $12850 \pm 5010$ \\
\hline distância $(\mu \mathrm{m})^{2,3,4}$ & $159 \pm 57$ & $215 \pm 58$ & $140 \pm 72$ \\
\hline \multicolumn{4}{|c|}{${ }^{1}$. diferença significativa entre todas as cepas $(p<0,05)$. } \\
\hline \multicolumn{4}{|c|}{ 2. diferença significativa entre as cepas CM e CMO $(p<0,05)$. } \\
\hline \multicolumn{4}{|c|}{${ }^{3}$. diferença significativa entre as cepas BE e CM $(p<0,05)$. } \\
\hline${ }^{4}$. diferença não significati & e as cepas BE & $(p>0,05$ & \\
\hline
\end{tabular}

\begin{tabular}{|c|c|c|c|}
\hline \multirow[t]{2}{*}{ Caracteres } & \multicolumn{3}{|c|}{ Cepas } \\
\hline & $\mathrm{BE}$ & CM & $\mathrm{CMO}$ \\
\hline \multicolumn{4}{|l|}{ Ovo uterino } \\
\hline área $\left(\mu \mathrm{m}^{2}\right)$ & $2730 \pm 625$ & $2882 \pm 767$ & $2887 \pm 592$ \\
\hline maior diâmetro $(\mu \mathrm{m})^{1}$ & $95 \pm 8$ & $103 \pm 11$ & $105 \pm 10$ \\
\hline menor diâmetro $(\mu \mathrm{m})$ & $35 \pm 6$ & $34 \pm 6$ & $34 \pm 7$ \\
\hline perímetro $(\mu \mathrm{m})$ & $245 \pm 27$ & $257 \pm 31$ & $257 \pm 22$ \\
\hline \multicolumn{4}{|l|}{ Espinho do ovo } \\
\hline área $\left(\mu \mathrm{m}^{2}\right)^{1}$ & $146 \pm 78$ & $114 \pm 68$ & $102 \pm 58$ \\
\hline maior diâmetro $(\mu \mathrm{m})$ & $19 \pm 6$ & $18 \pm 6$ & $16 \pm 5$ \\
\hline menor diâmetro $(\mu \mathrm{m})$ & $9 \pm 3$ & $7 \pm 2$ & $7 \pm 2$ \\
\hline perímetro $(\mu \mathrm{m})^{1}$ & $54 \pm 18$ & $49 \pm 18$ & $45 \pm 13$ \\
\hline \multicolumn{4}{|l|}{ Ventosas (área) } \\
\hline oral $\left(\mu m^{2}\right)^{1}$ & $2078 \pm 658$ & $2372 \pm 654$ & $1903 \pm 779$ \\
\hline ventral $\left(\mu \mathrm{m}^{2}\right)$ & $2205 \pm 784$ & $2398 \pm 771$ & $2097 \pm 667$ \\
\hline distância $(\mu \mathrm{m})$ & $144 \pm 31$ & $163 \pm 44$ & $158 \pm 58$ \\
\hline
\end{tabular}




\section{DISCUSSÃO}

O camundongo é o hospedeiro mais utilizado no estudo das características parasitológicas e morfológicas de cepas de S. mansoni, sobretudo porque é de fácil manutenção em laboratório, elimina ovos férteis nas fezes e os vermes adultos apresentam pleno desenvolvimento somático 293338 .

Bain \& Philipp 4 citam que um parasito utilizado em condições de laboratório descende de uma ou mais populações naturais com polimorfismo genético que, nessa nova condição, reduz a sua variabilidade. Como as cepas reduzem o polimorfismo genético, seria de se esperar que não ocorressem mudanças biológicas e morfológicas, ou ambas, nos vermes adultos.

Em hospedeiros permissivos, tais como o homem e camundongos, o início da oviposição ocorre em torno de 4-5 semanas após o contato com as cercárias. Através do oograma de tecidos intestinais demonstrou-se que, tanto em humanos ${ }^{46}$ quanto em camundongos ${ }^{43}$, os ovos produzidos pelas fêmeas passam por quatro estágios de maturação, até que sejam expelidos embrionados pelas fezes. Assim como os helmintos intestinais ${ }^{23}$, os vermes adultos de $S$. mansoni também apresentam um nicho de oviposição. Há evidências de que os sais biliares transportados pela veia porta estimulem a oviposição in vitro do S. mansoni . Em nosso experimento, todas as cepas apresentaram maior deposição de ovos no segmento distal do intestino delgado do que no intestino grosso $^{15}{ }^{31}$. Esta situação contrasta com estudos em primatas (inclusive o homem), onde tem se verificado que o sítio de oviposição é no intestino grosso. Por outro lado, a partir de um vasto estudo sobre a infecção esquistossomótica em casos autopsiados no Estado da Bahia (Brasil), concluiu-se que a forma clínica da doença afeta a distribuição topográfica dos ovos e dos vermes adultos de $S$. mansoni. Assim, a proporção de vermes e a concentração de ovos no colo transverso e porção média do jejuno foram maiores nos indivíduos com fibrose de Symmers do que nos indivíduos com formas menos graves $^{11}$.

Outro aspecto a ser destacado é que as cepas apresentaram diferenças na capacidade de migração no sistema porta como já descrito ${ }^{54}$. Entretanto, não se conhece o que determina esta diferença. A mudança do estado de hermafroditismo para sexos separados implicou no surgimento do canal ginecóforo, desenvolvimento acentuado de musculatura dos vermes machos e do sistema genital feminino, e redução da capacidade locomotora das fêmeas ${ }^{35} 45$. O papel dos adultos machos é, principalmente, transportar as fêmeas para o sítio de postura de ovos, que é facilitado por sua musculatura ${ }^{34} 35$. Curiosamente, a cepa CMO apresentou a menor capacidade de deslocamento e o menor número de ovos nas fezes. Em publicação anterior verificamos que o comprimento dos adultos machos era significativamente menor do que em outras cepas $^{29}$. Por outro lado, nesta cepa observamos um maior percentual de ovos viáveis. Um estudo desenvolvido a partir de um único casal de vermes poderia esclarecer se a redução de tamanho dos vermes adultos machos afeta a sua capacidade de migração e a oviposição das fêmeas.

A contagem de ovos nas fezes de camundongos tem sido utilizada para determinação do período prépatente da infecção esquistossomótica experimental e, mais raramente, para avaliar a cinética de eliminação de ovos na fase inicial da infecção ${ }^{46}$. Neste experimento, não foi confirmado que cepas apresentam diferenças no período pré-patente ${ }^{1418}$. Segundo alguns autores ${ }^{18}$, as cepas que são mantidas por mais tempo em laboratório apresentam o menor período pré-patente. No nosso caso, contudo, o menor período pré-patente foi da cepa mais recente mantida em laboratório (CM).

A dinâmica de eliminação de ovos nas fezes não tem sido avaliada nos estudos comparativos entre cepas brasileiras de $S$. mansoni. De acordo com os nossos resultados, as cepas apresentaram o mesmo comportamento. O padrão de eliminação foi semelhante ao descrito para a fase inicial de infecção: início com baixa contagem e um aumento progressivo (entre 49 e 72 dias pós-infecção) ${ }^{46}$. Estudos in vitro evidenciaram que a oviposição é caracterizada por três etapas: uma fase inicial onde as fêmeas produzem poucos ovos, um período de máxima oviposição e, finalmente, uma redução progressiva no número de ovos eliminados ${ }^{5}$. Isto ocorre cerca de três semanas após o período pré-patente ${ }^{51746}$. Neste experimento, a diminuição de oviposição não foi observada porque os exames parasitológicos foram realizados entre a sexta e a oitava semana de infecção.

A contagem de ovos é um indicador de fecundidade de vermes adultos mantidos in vivo e in vitro. Nestes ensaios foi demonstrado que há relação entre o número de ovos produzidos e a carga parasitária 251217 . No modelo murino, esta relação foi também observada na fase inicial da infecção esquistossomótica ${ }^{46}$. No presente experimento verificamos correlação positiva entre a produtividade de ovos (fezes e contagem de ovos no intestino) e o número de vermes adultos, apenas em uma das cepas (CMO). Entretanto, não é conhecido se este fator é variável entre cepas.

A taxa de maturação de cercárias em vermes adultos (infectividade) é também um critério aplicado na diferenciação de cepas. Não foi possível distinguir as cepas por este critério, embora os valores obtidos estejam de acordo com aqueles citados (entre 21 e $61 \%$ ) para outras cepas brasileiras ${ }^{155}$. Outros autores verificaram que uma cepa humana era mais infectante para camundongo do que uma outra de roedor, ambas do Vale do Rio Paraíba do Sul (São Paulo) ${ }^{6}$.

Os dados sobre a recuperação de vermes adultos demonstram que entre as cepas estudadas, a 
proporção foi semelhante entre machos e fêmeas (exceto na cepa $\mathrm{CMO}$ ). A origem desta desproporção tem sido exaustivamente estudada desde a década de 40 e não está esclarecida 274251 . Ao que parece, cada sexo apresenta distinta estratégia de transmissão: cercárias machos apresentam maior infectividade ${ }^{9} 57$ enquanto as fêmeas exibem maior longevidade ${ }^{9}$, em condições experimentais. Por outro lado, pode não haver predominância de um sexo (macho) e, mesmo assim, a infectividade ser máxima. Admite-se que nesta situação de equilíbrio haja uma interação entre os sexos, onde a presença do macho estimularia a infectividade das fêmeas ${ }^{7}$. Nossos dados fortalecem esta hipótese, embora sem diferença significativa, a maior infectividade foi observada em cepas cuja proporção entre macho e fêmea era de 1:1. Em hospedeiros permissivos, distintos genótipos do parasito poderiam se desenvolver, induzindo a uma acentuada competição entre as fêmeas e como conseqüência o aumento no número de machos ${ }^{8}$.

Neste experimento, as alterações morfológicas nos vermes adultos foram mais significativas do que os dados biológicos. Geralmente, o número de testículos e a distância entre as ventosas (em machos e fêmeas), o comprimento dos vermes adultos e o tamanho do ovo são utilizados como caracteres taxonômicos na descrição morfológica de cepas. O primeiro parâmetro varia segundo a cepa, independentemente de ser alopátrica ${ }^{33} 47$ ou simpátrica ${ }^{29} 30$. Recentemente, propusemos outros caracteres que foram empregados em cepas simpátricas. Diferenças significativas $(p<0,05)$ foram detectadas nos vermes de ambos os sexos: em todas as medidas dos ovos, espinhos e ventosas (fêmeas) e nos machos, o perímetro, eixos e diâmetro dos lobos testiculares ${ }^{37}$.

No presente trabalho foram utilizadas três cepas alopátricas mantidas durante várias gerações em laboratório e, mesmo assim, alguns caracteres eram diferentes entre todas as cepas ou entre duas delas. Assim, confirmamos que os vermes adultos machos de cepas mantidas por várias gerações em laboratório também apresentam diferenças morfológicas entre $\mathrm{si}^{29} 30$. Em recente publicação verificamos que os vermes adultos machos da cepa CMO apresentavam menor número de lobos testiculares e menor distância entre as ventosas do que outras cepas brasileiras (BH-Belo Horizonte, Minas Gerais e SJ-São José dos Campos, São Paulo) ${ }^{30}$. Os vermes adultos das cepas BE e CM foram pela primeira vez estudados no presente experimento.

O estudo dos lobos testiculares confirmou que alguns espécimes apresentavam lobos em posição anormal ou supranumerários 29303637 . O significado morfo-fisiológico desta estrutura é desconhecido: órgão reprodutivo funcional ou degenerado? De acordo com nossos estudos por microscopia de varredura laser confocal, os lóbulos supranumerários não apresentam as mesmas células ou espermatozóides dos lóbulos testiculares normais e nem a estes estão conectados ${ }^{32}$.

Poucos autores utilizaram os vermes adultos fêmeas nos estudos comparativos entre cepas ${ }^{19} 3341$. Os caracteres por outros autores analisados foram o comprimento do espécime, distância entre as ventosas e tamanho do ovo. $A$ análise destes dois últimos caracteres permitiu-lhes a distinção entre duas cepas (BH e SJ). Esta observação difere de nossos experimentos anteriores onde todos os caracteres do sistema reprodutor e das ventosas foram significativamente diferentes ${ }^{37}$.

As cepas estudadas neste experimento foram provenientes da mesma instituição com passagens seriadas e exclusivas em $B$. glabrata simpátrica e no camundongo albino. Portanto, todas sofreram a mesma pressão seletiva. Modificações induzidas pelo hospedeiro murino aparecem nas primeiras gerações das cepas em condições de laboratório 22 ou já na primeira passagem ${ }^{27}$. Conclui-se que as diferenças naturais (biológicas e morfológicas nos vermes adultos) das cepas são expressas, mesmo com a sua manutenção prolongada em condições de laboratório.

\section{AGRADECIMENTOS}

Ao excelente apoio técnico de Maria de Fátima Bastos Martins, Disciplina de Parasitologia, Universidade do Estado do Rio de Janeiro e a Dra. Lygia dos Reis Corrêa, Departamento de Malacologia, Instituto Oswaldo Cruz, pelo fornecimento das cepas.

\section{REFERÊNCIAS BIBLIOGRÁFICAS}

1. Anderson LA, Cheever AW. Comparison of geographical strains of Schistosoma mansoni in the mouse. Bulletin World Health Organization 46: 233- 242, 1972.

2. Andrade ZA, Sadigursky M. Um estudo comparativo das cepas Feira de Santana (Bahia) e Porto Rico do Schistosoma mansoni na infecção experimental do camundongo. Memórias do Instituto Oswaldo Cruz 80: 37-40, 1985.

3. Badr SG, Pica-Mattocia L, Moroni R, Angelico M, Cioli D. Effect of bile salts on oviposition in vitro by Schistosoma mansoni. Parasitology Research 85: 421-423, 1999.

4. Bain O, Philipp M. Animal models in the study of the phenomenon of parasitism: filariae and other parasites. Annales Parasitologie 66 (suppl 1):64-68, 1991.
5. Barth LR, Fernandes APM, Rodrigues V. Oviposition by Schistosoma mansoni during in vitro cultivation. Revista do Instituto de Medicina tropical São Paulo 38: 423-426, 1996.

6. Bastos OC, Magalhães LA, Pareja GB. Estudo da infecção esquistossomótica produzida pelas linhagens humana e silvestre do Vale do Rio Paraíba do Sul, SP (Brasil), em camundongos isogênicos. Revista de Saúde Pública de São Paulo 13: 335- 340, 1979.

7. Boissier J, Moné H. Experimental observations on the sex ratio of adult Schistosoma mansoni, with comments on the natural male bias. Parasitology 121: 379-383, 2000.

8. Boissier J, Moné H. Relationship between worm burden and male proportion in Schistosoma mansoni experimentally infected 
rodents and primates. A meta-analytical approach. International Journal for Parasitology 31: 1597-1599, 2001.

9. Boissier J, Morand S, Moné H. A review of performance and pathogenecity of male and female Schistosoma mansoni during the life-cycle. Parasitology 119: 447-454, 1999.

10. Cheever AW. Conditions affecting accuracy of potassium hydroxide digestion techniques for counting Schistosoma mansoni eggs in tissues. Bulletin World Health Organization 39: 328-331, 1968.

11. Cheever AW. A quantitative post-mortem study of schistosomiasis mansoni in man. American Journal of Tropical Medicine and Hygiene 17: 38-60, 1975.

12. Cheever AW, Mosimann JE, Deb S, Cheever EA, Duvall RH. Natural history of Schistosoma mansoni infection in mice: egg production, egg passage in the feces, and contribution of host and parasite death to changes in worm numbers. American Journal Tropical Medicine and Hygiene 50: 269- 280, 1994.

13. Combes C. Where do human schistosomes come from? An evolutionary approach. Trends in Ecology Evolution 5: 334337, 1990.

14. Conceição MJ, Coura JR. Comportamento experimental de amostras de Schistosoma mansoni em relação às formas clínicas de Esquistossomose. I- Estudo em camundongos. Memórias do Instituto Oswaldo Cruz 81: 61-66, 1986.

15. Costa MFFL, Katz N. Comparative studies of Schistosoma mansoni strains isolated from patientes with toxemic or intestinal forms of Schistossomiasis. American Journal of Tropical Medicine and Hygiene 31: 499-504, 1982.

16. Costa MFFL, Rocha RS, Katz N. Comparative study of Schistosoma mansoni isolated from patients with hepatosplenic and intestinal clinical forms of schistosomiasis. American Journal of Tropical Medicine and Hygiene 33: 918-923, 1984.

17. El-Ridi R, Ozaki T, Inaba T, Ito M, Kamiya M. Schistosoma mansoni oviposition in vitro reflects worm fecundity in vivo: individual-,parasite age- and host-dependent variations. International Journal for Parasitology 27: 381-387, 1997.

18. Fallon PG, Mubarak JS, Fookes RE, Niang M, Butterworth AE, Sturrock RF, Doenhoff MJ. Schistosoma mansoni: maturation rate and drug susceptibility of different geographic isolates. Experimental Parasitology 86: 29-36, 1997.

19. Figueiredo AC, Dias LCS, Marques EHF. Biological and morphological characteristics of Schistosoma mansoni from Ribeira Valley State of São Paulo, Brazil. I - Susceptibility of Biomphalaria tenagophila snail to sympatric S. mansoni strain. Revista do Instituto de Medicina Tropical de São Paulo 34: 199-203, 1992.

20. Fletcher M, Loverde PT, Woodruff DS. Genetic variation in Schistosoma mansoni: enzyme polymorphisms in populations from America, Southwest Asia, South America, and the West Indies. American Journal of Tropical Medicine and Hygiene 30: 406-421, 1981.

21. Frandsen F. Discussion of the relationship between Schistosoma and their intermediate hosts, assessment of the degree of host-parasite compatibility and evaluation of schistosome taxonomy. Zeitschrft fur Parasitenkunde 58: 275-296, 1979.

22. Higgins-Opitz SB, Dettman CD. The infection characteristics of a South African isolate of Schistosoma mansoni: a comparison with a Puerto Rican isolate in BALB/c mice and Mastomys coucha. Parasitology Research 77: 142-151, 1991.

23. Holmes JC. Competition, contacts, and other factors restricting niches of parasitic helminths. Annales du Parasitologie 65: 6972, 1990.

24. Kassim OO, Cheever AW, Richards CS. Schistosoma mansoni: Mice infected with different worm strains. Experimental Parasitology 48: 220-224, 1979.

25. Katz N, Chaves A, Pellegrino J. A simple device for quantitative stool thick-smear technique in schistosomiasis mansoni. Revista do Instituto de Medicina tropical de São Paulo 14: 397-400, 1972.

26. Kloetzel K. O problema das "raças" de S. mansoni. Hospital 56: 99-106, 1959.

27. Liberatos JD. Schistosoma mansoni: male-biased sex ratios snails and mice. Experimental Parasitology 64: 165-177, 1987.

28. Loverde PT, Dewald J, Minchella DJ, Bosshards SC, Damian RT. Evidence for host-induced selection in Schistosoma mansoni. Journal of Parasitology 71: 297-301, 1985.

29. Machado-Silva JR, Galvão C, Oliveira RMF, Presgrave OAF, Gomes DC. Schistosoma mansoni Sambom, 1907: Comparative morphological studies of same brazilian strains. Revista do Instituto de Medicina tropical de São Paulo 37: 441-447, 1995.

30. Machado-Silva JR, Galvão C, Presgrave OAF, Rey L, Gomes DC. Host-induced morphological changes of Schistosoma mansoni Sambon, 1907 male worms. Memórias do Instituto Oswaldo Cruz 89: 411-416, 1994.

31. Machado-Silva JR, Oliveira RMF, Rodrigues-Silva R, Maldonado Jr A, Rey L Roedores silvestres como modelos experimentais da esquistossomose mansônica: Akodon arviculoides (Rodentia; Cricetidae). Revista do Instituto de Medicina Tropical de São Paulo 33: 257-261, 1991.

32. Machado-Silva JR, Pelajo-Machado M, Lenzi HL, Gomes DC. Morphological study of adult male worms of Schistosoma mansoni Sambon, 1907 by confocal laser scanning microscopy. Memórias do Instituto Oswaldo Cruz 93 (suppl I): 303-307, 1998.

33. Magalhães LA, Carvalho JF. Estudo morfológico de Schistosoma mansoni pertencentes a linhagens de Belo Horizonte (MG) e de São José dos Campos (SP). Revista de Saúde Pública de São Paulo 7: 289-294, 1973.

34. Mair GR, Maule AG, Shaw R, Halton DW. Muscling in on parasitic flatworms. Parasitology Today 14: 73-76, 1998.

35. Morand S, Muller-Graf CDM. Muscles or testes? Comparative evidence for sexual competition among dioecious blood parasites (Schistosomatidae) of vertebrates. Parasitology 120: 45-56, 2000.

36. Najim AT. A male Schistosoma mansoni with two sets of testes. Journal of Parasitology 37: 545-546, 1951.

37. Neves RH, Pereira MJS, Oliveira RMF, Gomes DC, Machado-Silva JR. Schistosoma mansoni Sambon, 1907: Morphometric differences between adult worms from sympatric rodents and human isolates. Memórias do Instituto Oswaldo Cruz 93: 309-312, 1998.

38. Nyndo M, Farah IO. The baboon as a non-human primate model of schistosomiasis mansoni. Parasitology Today 15: 478-482, 1999.

39. Paraense WL, Corrêa LR Variation in susceptibility of populations of Australorbis glabratus to a strain Schistosoma mansoni. 
Revista do Instituto de Medicina Tropical de São Paulo 5: 15-22, 1963a.

40. Paraense WL, Corrêa LR. Susceptibility of Australorbis tenagophilus to infection with Schistosoma mansoni. Revista do Instituto de Medicina Tropical de São Paulo 5: 23-29, 1963b.

41. Paraense WL, Corrêa LR. Observations on two biological races of Schistosoma mansoni. Memórias do Instituto Oswaldo Cruz 76: 287-291, 1981.

42. Paraense WL, Santos JM. O sexo do Schistosoma mansoni em infestações produzidas por cercarias de um único molusco. Memórias do Instituto Oswaldo Cruz 47: 35-49, 1949.

43. Pellegrino J, Oliveira CA, Faria J, Cunha AS. New approaching to the screening of drugs in experimental schistosomiasis mansoni infection. American Journal of Tropical Medicine and Hygiene 11: 210-215, 1962.

44. Pitchford RJ. A check list of definitive hosts exhibiting evidence of the genus Schistosoma Weinland, 1858 acquired naturally in Africa and the Middle East. Journal of Helminthology 51: 229-252, 1979.

45. Platt TR, Brooks DR. Evolution of the schistosomes (Digenea: Schistosomatidae): the origin of dioecy and colonization of the venous system. Journal of Parasitology 83: 1035-1044, 1977.

46. Prata A. Biópsia retal na esquistossomose. Bases e aplicações no diagnóstico e tratamento. Tese, Serviço Nacional de Educação Sanitária, Rio de Janeiro, RJ, 1957.

47. Rocha RL, Rocha MOC, Pedroso ERP, Colosimo EA, Coelho PMZ. Egg excretion in the initial phase of experimental murine Schistosomiasis mansoni: stability and association with worm burden. Revista do Instituto de Medicina Tropical de São Paulo 37: 325-329, 1995.

48. Saoud MFA. Comparative studies on the characteristics of same geographical strains of Schistosoma mansoni in mice and hamsters. Journal of Helminthology 39: 101-112, 1965.

49. Siegel S. Estatística não paramétrica para as ciências do comportamento. Editora McGraw-Hill, Rio de Janeiro, 1975.
50. Smithers SR, Terry RJ. The infection of laboratory hosts with cercariae of Schistosoma mansoni and the recovery of the adults worms. Parasitology 55: 695-700, 1965.

51. Souza CP, Jannotti-Passos LK, Ferreira SS, Vieira IBF. Schistosoma mansoni: the sex ratios of worms in animals infected with cercariae from three species of Biomphalaria. Revista do Instituto de Medicina Tropical de São Paulo 38 : 141-145, 1996.

52. Snyder SD, Loker E. Evolutionary relationship among the schistosomatidae (Platyhelminths:Digenea) and an Asian origin for Schistosoma. Journal of Parasitology 86: 283-288, 2000.

53. Thiongo FW, Madsen H, Ouma JH, Andreassen J, Christensen NO. Host-parasite relationships in infections with two Kenyan isolates of Schistosoma mansoni in NMRI mice. Journal of Parasitology 83: 330-332, 1997.

54. Valadares TE, Coelho PMZ, Pellegrino J, Sampaio IBM. Schistosoma mansoni: Comparação da oviposição entre as cepas LE (Belo Horizonte), SP (São Paulo) e ST (Libéria) em camundongos. Revista do Instituto de Medicina Tropical de São Paulo 23: 1-5, 1981.

55. Warren KS. A comparison of Puerto Rican, Brazilian, Egyptian and Tanzanian strains of Schistosoma mansoni in mice: Penetration of cercariae maturation of schistosomes and production of liver disease. Transactions of the Royal Society Tropical Medicine and Hygiene 61: 795-802, 1967.

56. Working Committee for the Biological Characterization of Laboratory Animals/GV-SOLAS. Guidelines for specification of animals and husbandry methods when reporting the results of animal experiments. Laboratory Animals 19: 106-108, 1985.

57. Zanotti-Magalhães EM, Magalhães LA, Carvalho JF. Relação entre a patogenicidade de Schistosoma mansoni em camundongos e a suscetibilidade do molusco vetor. IInfecciosidade das cercárias e carga de vermes. Revista de Saúde Pública de São Paulo 25: 359-366, 1991. 